In a separate study, Jedd Wolchok at Memorial SloanKettering Cancer Center in New York and his colleagues, treated 86 people with advanced melanoma using two compounds: nivolumab, also a PD-1 inhibitor, and ipilimumab, an approved drug that blocks a checkpoint inhibitor called CTLA-4. Tumours shrank by at least half in $40 \%$ of the patients. N. Engl. J. Med. http://dx.doi. org/10.1056/NEJMoa1305133; http://dx.doi.org/10.1056/ NEJMoa1302369 (2013)

\section{ASTRONOMY}

\section{Trap holds protoplanet dust}

Dust particles spotted around a young star support an idea about how planets are born.

Planet formation is a paradox: according to standard theory, dust grains orbiting newborn stars should spiral into those stars rather than accrete to form planets. Astronomers have suggested that there are regions, or 'pressure bumps', where density and pressure gradients trap particles long enough to allow them to clump together.

A team led by Nienke van der Marel at Leiden University in the Netherlands has observed such a trap around the star Oph IRS 48 located about 120 parsecs from Earth. The Atacama Large Millimeter/submillimeter Array in Chile detected a crescent-shaped cluster on one side of the star - probably a reservoir of coalescing dust grains (pictured as an artist's impression).

Science 340, 1199-1202 (2013)

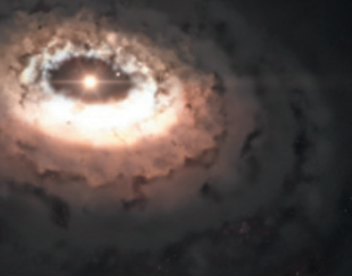

ENZYME ENGINEERING

\section{Serendipity outstrips design}

Accelerated evolution of an artificial enzyme improved its activity several-thousand fold, owing to unexpectedly extreme remodelling of its active site.

A team led by Donald

Hilvert and Nenad Ban at the Swiss Federal Institute of Technology in Zurich optimized a computationally designed enzyme with several rounds of random mutagenesis and screening. The activity levels of the evolving enzyme eventually approached those of natural enzymes, but the protein no longer catalysed its reaction using the machinery the researchers had intended. An amino-acid residue installed to help rearrange bonds was abandoned for one that emerged at another location in the active site. Such swaps could be important in natural-enzyme evolution and design efforts, the authors say. Nature Chem. Biol. http://dx.doi. org/10.1038/nchembio.1276 (2013)

\section{MOLECULAR BIOLOGY}

\section{Boosting plant defence}

The discovery of a gene that regulates the effects of the plant hormone jasmonic acid might lead to ways to increase pest resistance in crops, without hindering their growth.

Jasmonic acid helps plants to fend off insects and pathogens; it also regulates aspects of plant development, including fertility and fruit ripening. Daoxin Xie of Tsinghua University in Beijing and his colleagues identified a gene called JAV1 in the model plant Arabidopsis thaliana that suppresses several responses triggered by jasmonic acid. The JAV1 protein was degraded when insects or fungi attacked. Silencing JAV1 boosted plant resistance to disease, but had no adverse effect on fertility or other developmental processes. Mol. Cell 50, 506-517 (2013)

COMMUNITY CHOICE

The most viewed papers in science

\title{
One polymer with multiple forms
}

\section{HIGHLY READ \\ on pubs.acs.org in May \\ An unusual material can switch between polymers from two different classes with the addition of light.}

Da-Hui Qu, He Tian and their colleagues at the East China University of Science and Technology in Shanghai combined two types of molecules. Cyclodextrins form non-covalent complexes to yield supramolecular polymers, whereas coumarins form covalent bonds with each other under one wavelength of light, and release those bonds under another. The material that the researchers created could go from a supramolecular polymer to a covalent polymer and back again with the addition of light; adding a detergent produced a reversible hydrogel. Substances with switchable properties can combine advantages of distinct polymers in a single platform, the authors say.

Langmuir 29, 5345-5350 (2013)

\section{palaeontology \\ Big lizard among mammals Tomatoes make tubers}

A giant, plant-eating

lizard successfully competed with mammals about 40 million to 36 million years ago.

Researchers led by Jason Head at the University of Nebraska-Lincoln identified the lizard in a diverse assemblage of fossils collected in Myanmar. The teeth and jaws of the creature revealed that it was a plant-eater, and at an estimated 27 kilograms, it was one of the largest animals in the area. The researchers dubbed the species - which was almost twice the length of any living herbivorous lizard Barbaturex morrisoni after the singer Jim Morrison, who famously proclaimed himself the lizard king.

Reptiles need external heat to keep their bodies warm, so the hotter temperatures of past climates could have allowed the large lizards to survive, the authors say. Proc. R. Soc. B 280, 20130665 (2013)
Boosting levels of a hormone in tomato plants (Solanum lycopersicum) causes them to make tubers, like their sibling species the potato (Solanum tuberosum).

Yuval Eshed at the Weizmann Institute of Science in Rehovot, Israel, Eliezer Lifschitz at the Israel Institute of Technology in Haifa and their team engineered tomatoes to have high levels of a cytokinin, a type of hormone found in all plants. The tomatoes formed tiny tubers (pictured) at the base of leaves along their stems, where cells divide and levels of hormones fluctuate.

Giving potato plants the hormone in culture also elicited small spuds along plant stems. The authors suggest that a simple, common mechanism might prompt tubers in other species.

Curr. Biol. http://dx.doi. org/10.1016/j.cub.2013.04.061 (2013)

\section{$\rightarrow$ NATURE.COM}

For the latest research published by Naturevisit:

www.nature.com/latestresearch 